\title{
Primary Squamous Cell Carcinoma of the Stomach: Two Case Reports and a Literature Review
}

Yu-Ta Lin ${ }^{1}$, Yang-Yuan Chen ${ }^{1 *}$, Sung-Mao Tsai ${ }^{2}$, Jen-Wei Chou ${ }^{1}$, Li-Yuan Bai ${ }^{3}$, Ming-Yu Lien ${ }^{3}$ and Tsung-Wei Chen ${ }^{4}$

${ }^{1}$ Division of Gastroenterology, China Medical University Hospital, China Medical University, Taichung, Taiwan

${ }^{2}$ Division of Gastroenterology, Taichung Tzu Chi Hospital, Taiwan

${ }^{3}$ Division of Hematology/Oncology, China Medical University Hospital, China Medical University, Taichung, Taiwan

${ }^{4}$ Department of Pathology, China Medical University Hospital, Taichung, Taiwan

\begin{abstract}
Primary squamous cell carcinoma (SCC) of the stomach is rare. In this paper, the patient in Case 1 is a 57 -yearold man who underwent partial gastrectomy and adjuvant chemotherapy for primary SCC of the stomach. He survives more than 4 years as of the writing of this report. The patient in Case 2 is a 49 -year-old man who was also diagnosed as primary SCC of the stomach. He died of massive gastric cancer bleeding 2 months after the diagnosis. This report is followed by a literature review and further discussion of diagnostic criteria and palliative gastrectomy in these patients.
\end{abstract}

\section{Introduction}

Gastric cancer is one of the most common cancers worldwide. The vast majority of gastric cancer is adenocarcinoma, accounting for more than $90 \%$ of gastric malignancy. By contrast, primary squamous cell carcinoma (SCC) of the stomach is rare, with an incidence of $0.04-0.07 \%$ [1]. In this paper, we report 2 cases of primary SCC of the stomach.

\section{Case Report 1}

A 57-year-old man was admitted for a 2-week history of dysphagia to solids. He complained of a sensation of throat obstruction seconds after swallowing food. He had no difficulty swallowing liquids, and he experienced no coughing or choking during food consumption. He had smoked one pack of cigarettes daily for the past 30 years. Physical examination provided unremarkable findings. Furthermore, complete blood count and differential count as well as liver and renal function test results were all within normal limits. Esophagogastroduodenoscopy (EGD) showed a normal esophagus and esophagogastric (EG) junction; however, a large tumor with central ulceration was noted in the gastric cardia (Figure 1). Endoscopic ultrasound indicated a tumor measuring

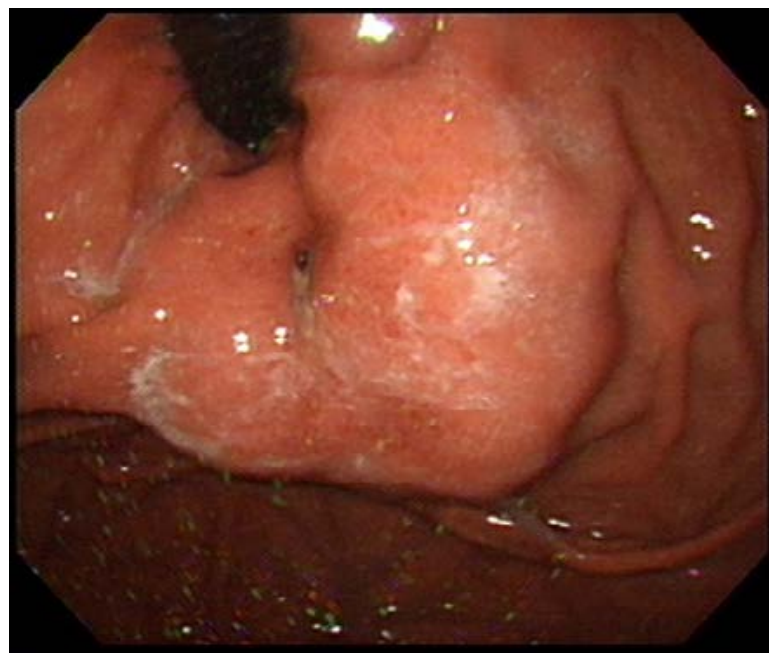

Figure 1: Upper GI endoscopy of the gastric cardia. The tumor was located in the gastric cardia.

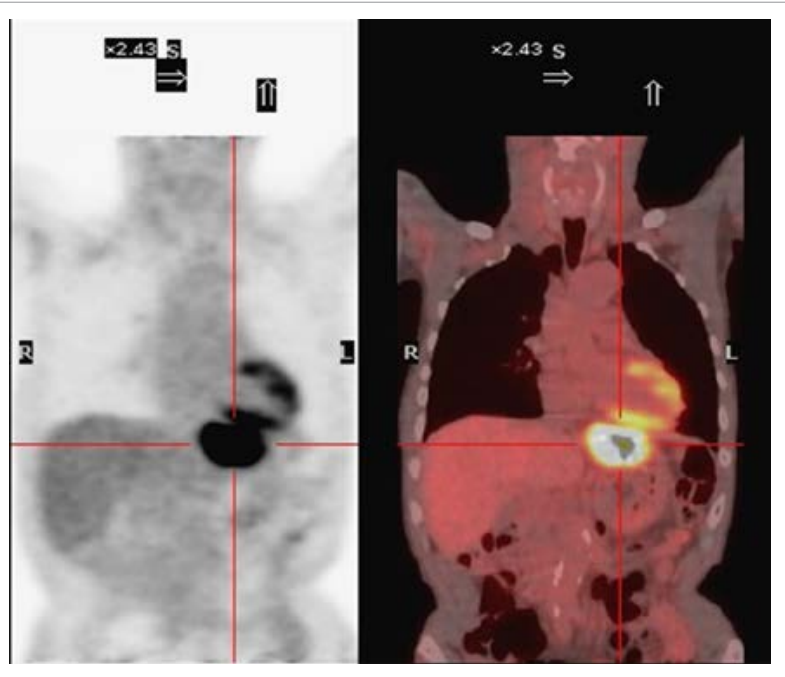

Figure 2: The PET-CT scan showed that radioactivity uptake occurred only in the stomach and excluded other primary malignancy origins.

$3.0 \times 4.2 \mathrm{~cm}$ and exhibiting heterogeneous echogenicity and a distinct margin. Biopsy of the central ulcerative area revealed moderately differentiated SCC. Abdominal computerized tomography (CT) indicated central necrosis within this gastric cancer but no evidence of distant metastasis. The patient was subjected to a positron emission tomography (PET) scan for surveying possible extra-GI tract primary malignancy, and the results demonstrated increased FDG radioactivity only in the gastric cardiac area (Figure 2). On the basis of a diagnosis

*Corresponding author: Yang-Yuan Chen, Division of Gastroenterology, China Medical University, China Medical University Hospital, 3 Lane 138 TaiAn 2nd Street Changhua, 500 Taiwan, China, Tel: 886-4-7359253; E-mail: ychen02@gmail.com

Received July 15, 2015; Accepted August 14, 2015; Published August 16, 2015.

Citation: Lin YT, Chen YY, Tsai SM, Chou JW, Bai LY, et al. (2015) Primary Squamous Cell Carcinoma of the Stomach: Two Case Reports and a Literature Review. J Cytol Histol 6: 353. doi:10.4172/2157-7099.1000353

Copyright: (C) 2015 Lin Y, et al. This is an open-access article distributed under the terms of the Creative Commons Attribution License, which permits unrestricted use, distribution, and reproduction in any medium, provided the original author and source are credited. 
Citation: Lin YT, Chen YY, Tsai SM, Chou JW, Bai LY, et al. (2015) Primary Squamous Cell Carcinoma of the Stomach: Two Case Reports and a Literature Review. J Cytol Histol 6: 353. doi:10.4172/2157-7099.1000353

of primary gastric cardia SCC, the patient subsequently underwent proximal gastrectomy. The resected specimen comprised one ulcerative tumor (measuring $4.5 \times 3.5 \times 3.0 \mathrm{~cm}$ ) with extragastric growth into the adjacent liver. Pathology revealed the occurrence of SCC without any adenocarcinomatous component and pathologic stage was T4N0M0. The mucosa over the esophagus and EG junction remained intact and free of tumor invasion (Figure 3). After surgery, adjuvant chemotherapy was administered with cisplatin $\left(70 \mathrm{mg} / \mathrm{m}^{2}\right.$ bolus infusion, Day 1$)$ and 5 -fluorouracil ( $900 \mathrm{mg} / \mathrm{m}^{2}$ bolus infusion, Days 1-4) from March 2011 to January 2013. Because of impaired renal function, the regimen was then shifted to carboplatin $\left(170 \mathrm{mg} / \mathrm{m}^{2}\right.$ bolus infusion, Day 1) and 5-fluorouracil (700 mg/m $\mathrm{m}^{2}$ bolus infusion, Days 1-4) from Feb 2013 to Apr 2015. The patient's disease progression subsided during chemotherapy and he is alive as of the writing of this report.

\section{Case Report 2}

A 49-year-old man was referred to our hospital for dysphagia and loss of body weight. Approximately 4 months previously, the patient noted mild dysphagia, particularly when swallowing solids. However, he was not concerned about the symptom because it was mild. Although he did not reduce his caloric intake, his body weight dropped from 50 to $45 \mathrm{~kg}$ in the following months. Physical examination showed no remarkable findings. Hemoglobin was $11.8 \mathrm{~g} / \mathrm{dL}$. White-cell and platelet counts, as well as liver and renal function test results were within normal limits. He underwent EGD, which revealed (1) a $2 \times 3 \mathrm{~cm}$ reddish flat mucosa without avascular area over the middle esophagus (Figure 4), and (2) a large tumor with central necrosis at the gastric cardia (Figure 5). The mucosa over the esophagus and EG junction remained intact and free of tumor invasion. Biopsy for the esophageal discoloration showed SCC in situ. The other biopsy for gastric lesion showed poorly differentiated SCC tumors, with immunohistochemical stains of $\mathrm{p} 40(+), \mathrm{CK} 5 / 6(+), \mathrm{CK} 7(-)$, and synaptophysin(-). CT for staging revealed a biggastric tumor $(9.6 \mathrm{~cm})$ in the cardiac portion; the results also indicated several enlarged lymph nodes in the perigastric area, military lesions in both lungs, and one hypovascular tumor $(6.6 \mathrm{~cm})$ in the section 8 of liver. PET scan showed increased FDG radioactivity at the gastric cardia, intraabdominal lymph nodes, lung nodules, hepatic lesion, skull, cervical vertebrae, thoracic vertebrae, and right femur. The PET scan could not identify a definite hypermetabolic lesion in the esophageal region. For advanced gastric SCC (T3N3M1) and esophageal SCC, palliative chemotherapy (cisplastin $50 \mathrm{mg} / \mathrm{m}^{2}$,

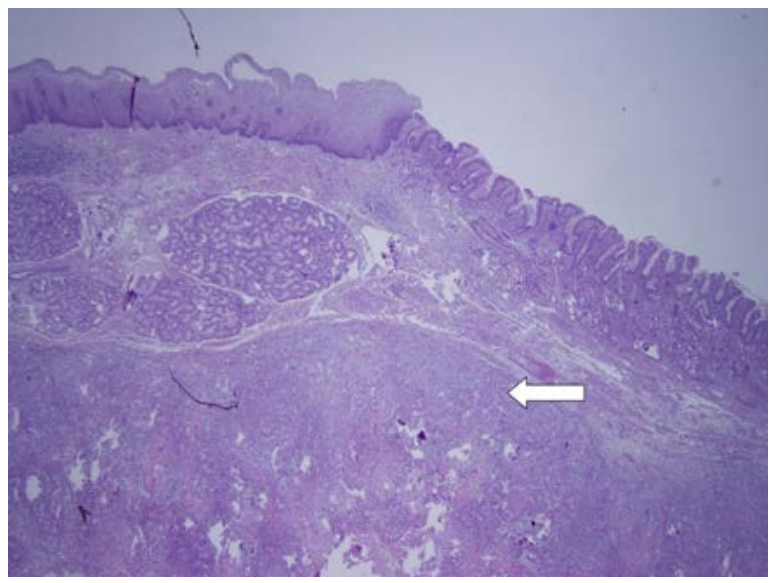

Figure 3: Microscopic examination of the gastric and esophageal junction (hematoxylin and eosin, 20X). The esophagus epithelium was normal with the tumor predominantly located in the gastric cardia (arrow).

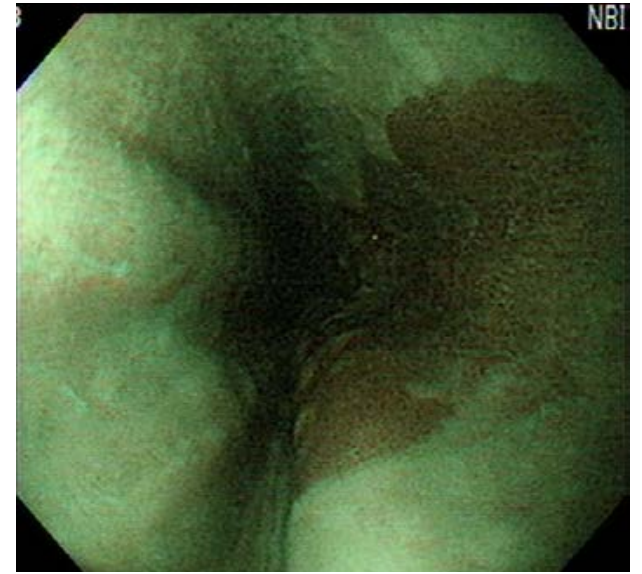

Figure 4: For reddish flat mucosa (white light imaging) over the middle esophagus, when using narrow band imaging endoscopy, it could be recognized as a clear brownish area.

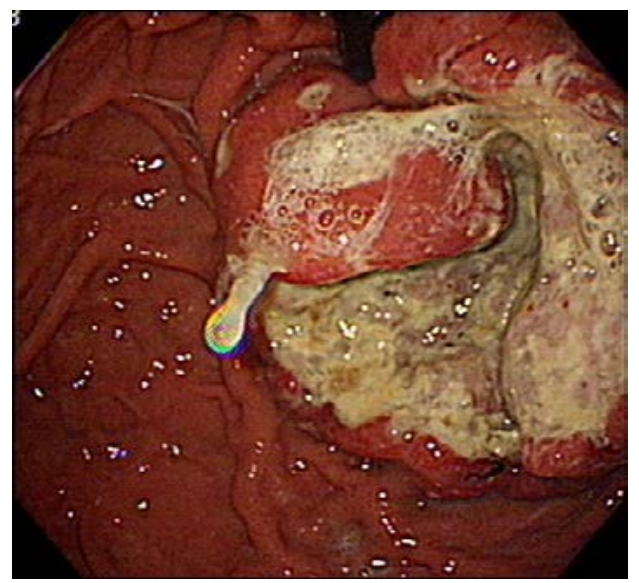

Figure 5: In the Case 2, there was a large tumor with central necrosis at the gastric cardia.

5-Fluorouracil $200 \mathrm{mg} / \mathrm{m}^{2}$ ) followed by concurrent chemoradiotherapy (cisplatin $30 \mathrm{mg} / \mathrm{m}^{2}, 3000 \mathrm{cGy} / 12 \mathrm{Fx}$ ) were administered. However, the patient died of massive gastric cancer bleeding 2 months after the initiation of chemotherapy.

\section{Discussion}

Gastric cancer is a major health problem worldwide. Adenocarcinoma is the most common type of gastric cancer, accounting for more than $90 \%$ of gastric malignancy. Remnants are lymphomas, gastrointestinal stromal tumors, carcinoid tumors, metastatic diseases, and primary SCC. Rörig first reported primary SCC of the stomach in 1895 [2], but to date, fewer than 100 cases have been reported in the English language literature. Primary gastric SCC is extremely rare, with an estimated incidence of $0.04-0.07 \% .^{-1}$ Men have a higher likelihood of developing primary gastric SCC compared with women, with a male-to-female ratio of 3.5:1. The peak incidence of primary gastric SCC is in the sixth decade of life. The most common tumor location is the proximal third of the stomach. Diagnosed tumors have a mean diameter of $7 \mathrm{~cm}[3,4]$.

In contrast to gastric adenocarcinoma arising from the glandular epithelium of the gastric mucosa, the pathogenesis of gastric SCC is not clearly understood. Several theories have been proposed regarding the 
Citation: Lin YT, Chen YY, Tsai SM, Chou JW, Bai LY, et al. (2015) Primary Squamous Cell Carcinoma of the Stomach: Two Case Reports and a Literature Review. J Cytol Histol 6: 353. doi:10.4172/2157-7099.1000353

pathogenesis of gastric SCC, including (1) ectopic squamous cells in the gastric mucosa, (2) squamous metaplasia of the gastric mucosa before malignant transformation, (3) totipotential stem cell in the gastric mucosa, (4) squamous differentiation in a preexisting adenocarcinoma, and (5) gastric vascular endothelial cells [5].

Because squamous epithelium lines the esophagus rather than the stomach, SCC involving both the gastric cardia and distal esophagus exhibits high possibility of an esophageal origin. Parks in 1967 suggested 3 diagnostic criteria for primary SCC of the stomach [6]: (1) The tumor must not extend into the esophagus; (2) the tumor must not be located in the cardia; and (3) there must be no evidence of SCC in any other organ. A debatable point is that, according to Parks' criteria, a true primary gastric SCC might be excluded if the tumor is located in the cardia. To obtain a precise diagnosis in primary gastric SCC, obtaining and integrating all clinical clues, including pathology, laboratory data, and image studies, is imperative. However, a tumor's location in the gastric cardia should not be designated as an exclusion condition. Instead of adhering to Parks' criteria, we could adopt the Japanese Gastric Cancer Association criteria: (1) all tumor cells are SCC cells, without adenocarcinomatous components in any section, and (2) distinct evidence that SCC arises directly from the gastric mucosa [7].

Immunohistochemicalstry are typically adopted to confirm the diagnosis of SCC, particularly strong staining for CK5/6, p40, and p63 [8-11]. However, immunohistochemistry is not applicable in discriminating primary SCCs from different tumor sites, although it may suggest the most likely site in most cases with adenocarcinoma of unknown primary origin [10].

Metastatic SCC of the stomach mainly develops via the lymphatic route rather than the bloodstream route. The most prevalent primary sites are the lung, breast, skin, and esophagus [12]. PET scan is currently one of the most rigorous methods and provides useful information for detecting suspicious metastasis. In Case 1 of the present study, the PET scan demonstrated no other organs with increased FDG radioactivity except the stomach. In Case 2, although the tissue biopsy from the middle esophagus showed SCC in situ, no increase in FDG radioactivity was observed in the esophagus. This is possibly because signals from esophageal SCC were too weak to be detected. Thus, for patient in Case 2 , primary gastric SCC is the most likely diagnosis because the size of gastric cardial cancer is out of proportion with others; in situ SCC of the esophagus is considered to be a second primary cancer.

The prognosis of gastric SCC is contradictory. Some reports have indicated that primary gastric SCC has a more favorable prognosis compared with gastric adenocarcinoma [2,13], whereas others have suggested the opposite [3-5]. The overall survival of patients with gastric SCC is from 7 months to 8 years. The patient in Case 1 (with adjacent liver involvement) survives for more than 4 years as of the writing of this report, whereas that in Case 2 died of massive tumor bleeding; therefore, we conclude that palliative gastrectomy or tumor resection for patients with advanced gastric SCC appears to provide symptomatic relief of pain, bleeding, obstruction, and possibly prolong survival [14-16].

Both patients in this study received combination chemotherapy with cisplatin plus 5-fluorouracil. No standard chemotherapy or chemotherapeutic regimens are established for this disease. Numerous case series are required for deriving precise information.

\section{References}

1. Straus R, Heschel S, Fortmann DJ (1969) Primary adenosquamous carcinoma of the stomach. A case report and review. Cancer 24: 985-995.

2. Altshuler JH, Shaka JA (1966) Squamous cell carcinoma of the stomach Review of the literature and report of a case. Cancer 19: 831-838.

3. Wakabayashi H, Matsutani T, Fujita I, Kanazawa Y, Nomura T, et al. (2014) A rare case of primary squamous cell carcinoma of the stomach and a review of the 56 cases reported in Japan. J Gastric Cancer 14: 58-62.

4. Hwang SH, Lee JH, Kim K, Shin DH, Kim JY, et al. (2014) Primary squamous cell carcinoma of the stomach: A case report. Oncol Lett 8: 2122-2124.

5. Callacondo D, Ganoza-Salas A, Anicama-Lima W, Quispe-Mauricio A Longacre TA (2009) Primary squamous cell carcinoma of the stomach with paraneoplastic leukocytosis: a case report and review of literature. Hum Pathol 40: 1494-1498.

6. Parks RE (1967) Squamous neoplasms of the stomach. Am J Roentgenol Radium Ther Nucl Med 101: 447-449.

7. Japanese Gastric Cancer A (1999) Japanese classification of gastric carcinoma (13thedn.) Kanchara, Tokyo.

8. Akbulut S, Finci R, Ozyilkan E (2003) Primary squamous cell carcinoma of the stomach: a case report. Acta Gastroenterol Belg 66: 189-190.

9. Wang G, Chen P, Shi L, Zhao W1 (2014) Squamous cell carcinoma arising from an unknown primary site metastasizing to the stomach: A case report. Oncol Lett 7: 1063-1066.

10. Hu JB, Zhu YH, Jin M, Sun XN (2013) Gastric and duodenal squamous cell carcinoma: metastatic or primary? World J Surg Oncol 11: 204.

11. Kanthan R, Senger JL, Diudea D, Kanthan S (2011) A review of duodena metastases from squamous cell carcinoma of the cervix presenting as an upper gastrointestinal bleed. World J Surg Oncol 9: 113.

12. Sarangapani A, Kini R, Chitra S, Pugazhendhi T, Jeevan Kumar S (2011) Intramural metastatic squamous cell carcinoma of stomach. Indian J Surg 73 307-308.

13. Bonnheim DC, Sarac OK, Fett W (1985) Primary squamous cell carcinoma of the stomach. Am J Gastroenterol 80: 91-94.

14. Callacondo D, Ganoza-Salas A, Anicama-Lima W, Quispe-Mauricio A, Longacre TA (2009) Primary squamous cell carcinoma of the stomach with paraneoplastic leukocytosis: a case report and review of literature. Hum Pathol 40: $1494-1498$.

15. Schmidt C, Schmid A, Lüttges JE, Kremer B, Henne-Bruns D (2001) Primary squamous cell carcinoma of the stomach. Report of a case and review of literature. Hepatogastroenterology 48: 1033-1036.

16. Wang G, Chen P, Shi L, Zhao W (2014) Squamous cell carcinoma arising from an unknown primary site metastasizing to the stomach: A case report. Oncol Lett 7: 1063-1066. 